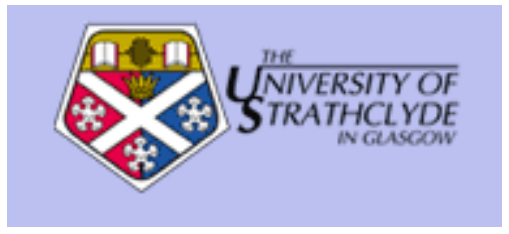

Torres-Sanchez, Carmen and Corney, Jonathan R. (2009) Towards functionally graded cellular microstructures. Journal of Mechanical Design , 131 (9). 091011-091018. ISSN 0094-2405

http://strathprints.strath.ac.uk/13936/

This is an author produced version of a paper published in Journal of Mechanical Design , 131 (9). 091011-091018. ISSN 0094-2405. This version has been peer-reviewed but does not include the final publisher proof corrections, published layout or pagination.

Strathprints is designed to allow users to access the research output of the University of Strathclyde. Copyright (C) and Moral Rights for the papers on this site are retained by the individual authors and/or other copyright owners. You may not engage in further distribution of the material for any profitmaking activities or any commercial gain. You may freely distribute both the url (http://strathprints.strath.ac.uk) and the content of this paper for research or study, educational, or not-for-profit purposes without prior permission or charge. You may freely distribute the url (http://strathprints.strath.ac.uk) of the Strathprints website.

Any correspondence concerning this service should be sent to The Strathprints Administrator: eprints@cis.strath.ac.uk 


\title{
Towards Functionally Graded Cellular Microstructures
}

\author{
Carmen Torres-Sanchez ${ }^{*}$ \\ Jonathan R. Corney \\ DMEM, University of Strathclyde, James Weir Building, Glasgow, United Kingdom, G1 1XJ
}

\begin{abstract}
Many materials require functionally graded cellular microstructures whose porosity (i.e. ratio of the void to solid volume of a material) is engineered to meet specific requirements. Indeed numerous applications have demonstrated the engineering potential of porous materials (e.g. polymeric foams) in areas ranging from biomaterial science through to structural engineering.
\end{abstract}

Although a huge variety of foams can be manufactured with homogeneous porosity, for heterogeneous foams there are no generic processes for controlling the distribution of porosity throughout the resulting matrix. Motivated by the desire to create a flexible process for engineering heterogeneous foams, this paper reports how ultrasound, applied during some of the foaming stages of a polyurethane (PU) melt, affects both the cellular structure and distribution of the pore size.

The experimental results allowed an empirical understanding of how the parameters of ultrasound exposure (i.e. frequency and acoustic pressure) influenced the volume and distribution of pores within the final polyurethane matrix: the data demonstrates that porosity (i.e. volume fraction) varies in direct proportion to the acoustic pressure magnitude of the ultrasound signal. The effects of ultrasound on porosity demonstrated by this work offer the prospect of a manufacturing process that can adjust the cellular geometry of foam and hence ensure that the resulting characteristics match the functional requirements.

Keywords: polymeric foam; graded porosity; sonication; manufacture

\section{INTRODUCTION}

It has been long recognized that the engineering performance of materials can be dramatically improved if their composition and structure is varied to match precisely the functional requirements. Optimum performance and synergetic features of manufactured engineering components could be achieved if the local porosity of foamed materials could be controlled [1]. Mechanical, electrical, thermal and structural properties are directly linked to density distribution (i.e. content and location of voids) [2;3]. The need for heterogeneous cellular materials has been widely recognised [4; 5]. Such heterogeneous materials have engineered gradients of composition or structure which offer superior performance over traditional homogeneous materials. Indeed, frequently heterogeneous materials demonstrate dramatic synergy (e.g. biological materials such as bone, tooth, shell, spider silk, etc) [6; 7].

The enormous difficulties of designing and forming such complex materials using traditional manufacturing methods has so far prevented their widespread use in engineering applications $[8 ; 9]$. Despite the need for heterogeneous materials (in fields such as thermal and microelectronic technology, filtration, drug release, tissue engineering, and biomaterial fabrication of scaffolds and orthopaedic implants) the digital technologies that support the design and manufacture of these components are only nascent.

So while advances in digital technologies for 3D modelling and manufacturing technologies (CAD/CAM) have been remarkable [10], there are few viable methods for translating digital representations of heterogeneous materials into physical objects with gradients of composition, structure and resulting physical properties [11].

A polymeric foam is a particular example of a heterogeneous material, since it is composed of at least two phases, one (or more) solid, plus voids whose size and distribution can be varied. Polymeric foam materials have demonstrated great application potential in a myriad of fields (biomaterials, tissue engineering, structural mechanics, etc) because of their lightness, low density, chemical inertness, high wear resistance, thermal and acoustic insulation [12]. This kind of versatility makes foam exceptional as a design material. Moreover, they have compositional similarities with natural bone and, some of them, a certain level of bioresorbability. Foam core materials offer weight minimisation, and the possibility of being blended with ceramic, or metal, to form polymer-ceramic/metal composites that overcome the disadvantages of a pure polymeric foam artefact (e.g. poor mechanical strength, short-lived nature, rapid degradability, etc).

The structure of a foam is characterised by the distribution, size and wall thickness of cells in the bulk material. These features are the result of many factors (e.g. temperature, pressure, reactants concentrations, etc) some of which are known to be affected by ultrasonic irradiation.

The aim of this paper is to report work which has demonstrated that the suitable manipulation of the position of the foaming polymeric matrix within a controlled sonicated field (i.e. with known acoustic pressure amplitude) permits the tailoring of the bubbles (i.e. cells) to a desired size (Figure 1). In other words, polymeric melts irradiated with ultrasound of variable intensity at critical points during the foaming process lead to a solid porous material with an engineered cellular structure [13].

This paper is structured as follows: After reviewing the literature concerning foam chemistry, ultrasound and sonochemistry (Background), the paper introduces the experimental procedure for a series of experiments performed to investigate the effect of an ultrasonic field on a vessel filled with PU foaming reactants (Methodology) and reports the strategy of characterisation of porosity gradation in the irradiated foams. The following section (Results) presents the comparison made between experimental and simulated results for the evaluation of the impact of the acoustic pressure on the porosity gradation within the foam cellular structure. An appraisal of this technique as a manufacturing technology for foams with a tailored porosity distribution is 
discussed in the final section before some conclusions are drawn on the wider significance of the findings.

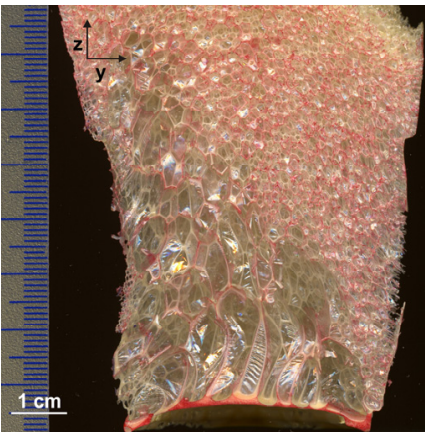

(a)

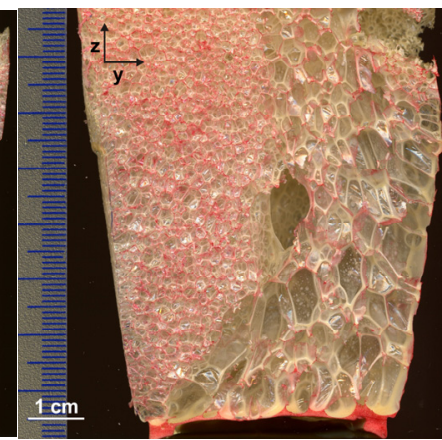

(b)
Figure 1: Cross-section of two foams sonicated at different positions in an ultrasonic standing wave (irradiating source was located on the left of these cross-sections)

\section{BACKGROUND}

\subsection{POLYMERIC FOAMS}

Foam is the dispersion of a gas in a liquid, which creates a characteristic structure when the matrix solidifies. Once cured, the foam consists of individual cells, or pores, the walls of which have completely polymerised and solidified to form a skeletal structure. For some polymeric foams, there might exist a latter stage at which those walls break, leaving an open structure of interconnected pores (flexible complexion). However, the polyurethane formulation used in this study was such that produced a final close-celled structure after curing (rigid structure) [14]. The chemical reaction that occurs between polyols and diisocyanate group to produce polyurethane $[15 ; 16]$ with distilled water employed as a blowing agent is:

$$
\begin{gathered}
\mathrm{HO}-\mathrm{R}-\mathrm{OH}(\text { polyol })+\mathrm{O}=\mathrm{C}=\mathrm{N}-\mathrm{R}^{\prime}-\mathrm{N}=\mathrm{C}=\mathrm{O}(\text { diisocyanate group }) \\
\rightarrow-\mathrm{O}-\mathrm{R}-\mathrm{O}-\mathrm{CO}-\mathrm{NH}-\mathrm{R}^{\prime}-\mathrm{NH}-\mathrm{CO}-(\mathbf{P U})+\mathrm{CO}_{2}(\text { gas })
\end{gathered}
$$

The water diffuses between the chains of polyurethane (PU) reacting at the same time with the isocyanate groups at the end of the chains, causing the reticulation, or cross-linking, of the polymer, and forming a rigid solid.

\subsection{ULTRASOUND AS A POROSITY-TAILORING AGENT}

Literature has widely reported ultrasonic irradiation to foams under a myriad of specific applications. Examples are the interaction ultrasound-foam enabled defoaming in bottling of fizzy drinks and the dissipation of foam in reaction and fermentation vessels $[17 ; 18]$, controlled polymerisation rate [19], assisted in the removal of contaminants [20], aided food dehydration [21] and drug delivery [22]. Many of these applications exploit the ultrasonically stimulated transientcavitation effect (rapid growth and explosive collapse of microscopic bubbles subjected to compression and rarefaction due to the irradiated soundwave passing through the liquid). An established research trend focusing on irradiation of foams under stable-cavitation conditions (i.e. rectified diffusion that enlarges the size of the bubble in a sustainable way) has not been found in the literature.
Under conditions of stable-cavitation, when bubbles of initial small radii suffer alternate expansion/contraction due to the sinusoidal soundwave, they increase their volume. Expansions are bigger than contractions and the bubble growth is in resonance with the soundwave and sustained in time. Bubble dynamics play an important role in pore enlargement, but other processes also enhanced by ultrasound (i.e. diffusion and mixing) will influence the dynamics of the process of foam formation. Particularly important in the context of foams and other high viscosity fluids is the ability of ultrasound to produce an increase in mass transport due to diffusion variation [23].

Essentially, sound affects the viscosity of fluids significantly (usually decreasing their viscosity), so acoustic radiation reduces the diffusion boundary layer, increases the concentration gradient and may increase the diffusion coefficient. In addition, turbulent convection provoked by ultrasound decreases the thickness of the mass transfer boundary layer, i.e. the wall of the pore, and increases transport through the membrane. However, if the shear forces provoked by ultrasound are excessive, some cells might rupture affecting the viscoelastic equilibrium in the matrix and, in extreme conditions, leading to a foam collapse (effect of transient-cavitation)

\section{METHODOLOGY}

To enable a systematic investigation of the effect of ultrasound on the formation and final porosity distribution of polyurethane foam, samples were irradiated in a temperature controlled (313K $\pm 1 \mathrm{~K})$ water bath over a fixed value of frequency and acoustic pressure. The schematic shown in Figure 2 illustrates the ultrasonic source and the polypropylene (material chosen for its similar acoustic impedance to water) container $(5 \mathrm{~cm}$ diameter, $7 \mathrm{~cm}$ height, $0.16 \mathrm{~mm}$ thickness) that held the reactants within the water bath and whose walls were lined with a PE air-bubble layer to minimise ultrasonic reflection. The use of water bath ensured the temperature of the environment could be controlled independently of the effects of ultrasound. The container was firmly clamped with a lab stand and positioned along the longitudinal axis of the bath. The ultrasonic sonotrodes sources used were a $20 \mathrm{kHz}$ Bandelin Sonopuls sonotrode, Germany, UW 3200 and a 25 or $30 \mathrm{kHz}$ Coltene Biosonic US100, USA. In order to have both sonotrode and receiver aligned, the sonotrode tip was immersed $2 \mathrm{~cm}$ below the free surface (i.e. sonication plane on ' $x z$ ' axis) and on the same plane that the central one of the container in 'xy' axis.

The reactants used in this study (Dow Europe GmbH, Switzerland) were pre-treated and the diisocyanate content in the mixture was rectified to ensure a repeatable and fixed $40 \%$ at the start of the polymerization. The amount of distilled water added was directly related to that amount $\left(20 \% \mathrm{vol} \mathrm{H}_{2} \mathrm{O}\right.$ per ml mixture $)$. This was done using the same procedure of stirring for a standard time of 70 seconds and minimising air intake into the mixture. All mixtures were sonicated in an open-vessel container to avoid the build up of the internal pressure due to the water vapour and gases (e.g. $\mathrm{CO}_{2}$ ) generated by the reaction that could provoke unwanted implosion of bubbles. Containers faced perpendicularly to the sonicating probe and had the opposite $180^{\circ}$ of their surface shielded by absorbent material to minimise reflections from the walls. This enabled an investigation of the effects of "direct" "near field' sonication. Thermocouples were held in the middle of the mixture and used to monitor the reaction and establish its completion (i.e. after peak temperature). 
The vessel was placed inside the bath in the plane of the sine wave (detected by the hydrophone) irradiated from the sonotrode. The 20-minute irradiation period was an off/on cycle of $2 \mathrm{~min}$ on/1 min off starting after adding the distilled water, and then left in the bath for 30 minutes until the foam was rigid. This cyclic irradiation was established by initial experimentation as sufficient to induce changes in the foam structure without causing collapse. Prior to this, the acoustic field in the bath had been accurately mapped so that the acoustic pressure conditions within the foam container were known (Figure 3). The ultrasonic irradiation characteristics were established by previous mapping of the ultrasonic bath using a needle-type hydrophone (Brüel\&Kjær,
Denmark, type 8103) shielded with a barrier made of the same open-vessel material for representative values.

The procedure followed is summarized as follows: 1. A measured amount of reactant was placed in the container located at a certain distance from the sonotrode; 2 . The process was initiated by addition of water (the chemical blowing agent and catalyst for the reaction); 3. Ultrasound of known acoustic pressure value was applied; 4. On completion of the reaction, the foam was left to cure for 48hours; 5 . Once the sonicated foams were fully cured, they were de-moulded and cut in half with a coarse-tooth saw and the cross-sections scanned for further analysis.

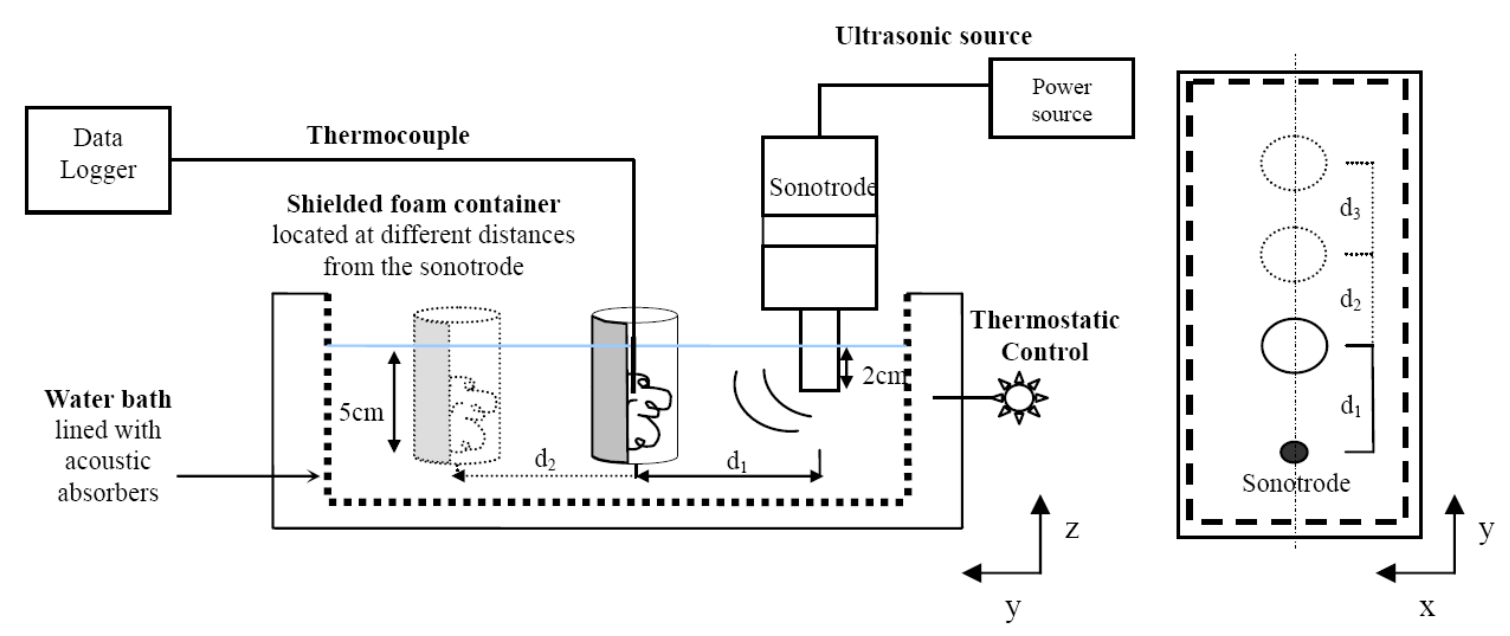

Figure 2: Schematic of the experimental rig, lateral and plan views, showing variable positions of a single foam container

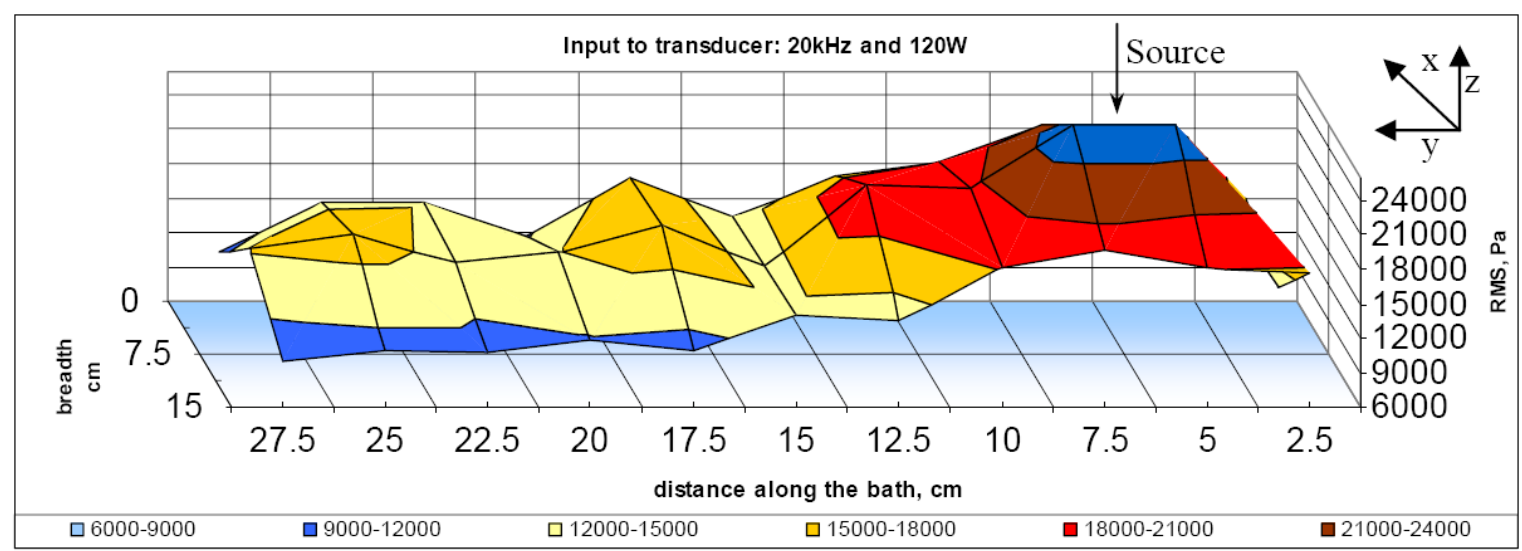

Figure 3: Mapping of water bath showing attenuation and partial maxima at half-wavelengths on the sonication plane

\subsection{QUANTIFYING POROSITY DISTRIBUTION IN PU FOAMS}

To assess the effects of the ultrasound exposure on the foam's cellular structure, a method of characterising the porosity distribution within a material is essential. For open-cell structures (e.g. flexible foams, rocks), porosity can be measured using liquid displacement techniques (e.g. Arquimedes', toluene infiltration displacement, mercury-porosimetry), which provide an average density value for the bulk material (e.g. measurement permeability and tortuosity in a sample). However, for this work, closed-pore foams were manufactured and these methods were not applicable. The lack of a systematic method to assess a heterogeneous material's porosity [24], was a difficulty for a direct assessment of the cellular structure in the irradiated foams. To overcome this obstacle an image processing application, known as 'Topo-porosity mapping' tool, was developed in MATLAB $^{\mathrm{TM}}$ to allow analysis and delineation of the foam porosity. This strategy considered the density of a cellular solid as the ratio of the density of the foam $(\rho *)$ to the density of the solid $\left(\rho_{\mathrm{s}}\right)$ material $\left(\rho * / \rho_{\mathrm{s}}\right)[12]$. The density of a foam is indicative of its 
porosity. Each sample was cut in half along its ' $x z$ ' axis and the porosity assessed using digital image analysis. Similar structure characterisation methods have been already used in aqueous and polymeric foams [25]. Within the sliced samples, the 3D network of the foam structure can be clearly observed (Figure 1). The samples were scanned at $1500 \mathrm{dpi}$ resolution in an EPSON

In essence, the program calculated the amount of cell wall material in different areas of a cross section of the foam. Points with the same range of porosity were connected by curves in the same way that contour lines in a topographic map connects continuous points of the same altitude. These topographic maps of porosity provided information on the porosity distribution within a foam cross-section, indicating the relative positions of areas with equivalent porosity (Figure 4).

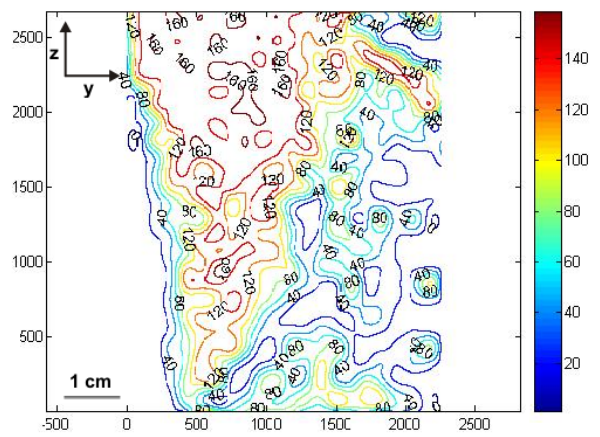

Figure 4: Contour lines connecting points of equal density of material in the cross-section of sonicated foam in figure 1(b)

In order to isolate the surface plane, the RGB values for colour of the foam matrix were filtered from the image. Colour power, colour threshold and intensity were also used to enhance the surface. Using this filtered image, a grid was applied to the image, which counted the pixels and adapted (i.e. reduced or expanded) the size of squares in the grid until they matched a
Perfection Scanner 1640SU. The purpose of the 'Topo-porosity mapping' tool was to correlate the topographic distribution of isolines of density in each sample with the manufacturing process parameters present during its formation (e.g. sonicating irradiation, frequency and relative position in the acoustic field)

given value of intensity. This intensity was set via the mesh spacing initially chosen so it reflected the observed distribution of cellular porosity. The image was then pixelated, so each grid contained a value which was the number of pixels contained in that area. Applying the "contour" option, a set of isoline curves was obtained and that connected all points of equal number of pixels, which was indirectly related to porosity and directly linked to density. The results were topographic pictures, where points of equal porosity are joined by contour lines. The relative position of the contour lines gave information about the rate of change within an image. Based on the ratio $\rho * / \rho_{\mathrm{s}}$, the contours effectively mapped porosity distribution where a value of 200 was set to be equivalent to the density of solid polyurethane. For areas where the colour was red, the density was higher, so porosity was low. For areas where colour was blue, porosity was higher. For areas with no lines, or spaces between lines, there was no variation of porosity in the samples (given the interval used to generate the plot). For example, when foam occupied $80 \%$ of the total volume, the value of the lines was 160 , as shown in Figure $5 \mathrm{c}$ and $\mathrm{d}$. By using the same parameters for colour filters and threshold, a comparative study among samples could be made. A cross-section of the topographic profile was created by recording values parallel to a $\mathrm{z}$-axis which was aligned to the irradiation plane of sonication (i.e. aligned with the horn tip). This allowed comparison of porosity gradient and sound pressure levels extracted from the simulator (Figure 6c). A validation of this technique was performed via comparison of the 'Topo-porosity' results versus direct measurements of porosity on the sample. The description of the procedure and the results can be found elsewhere [26].
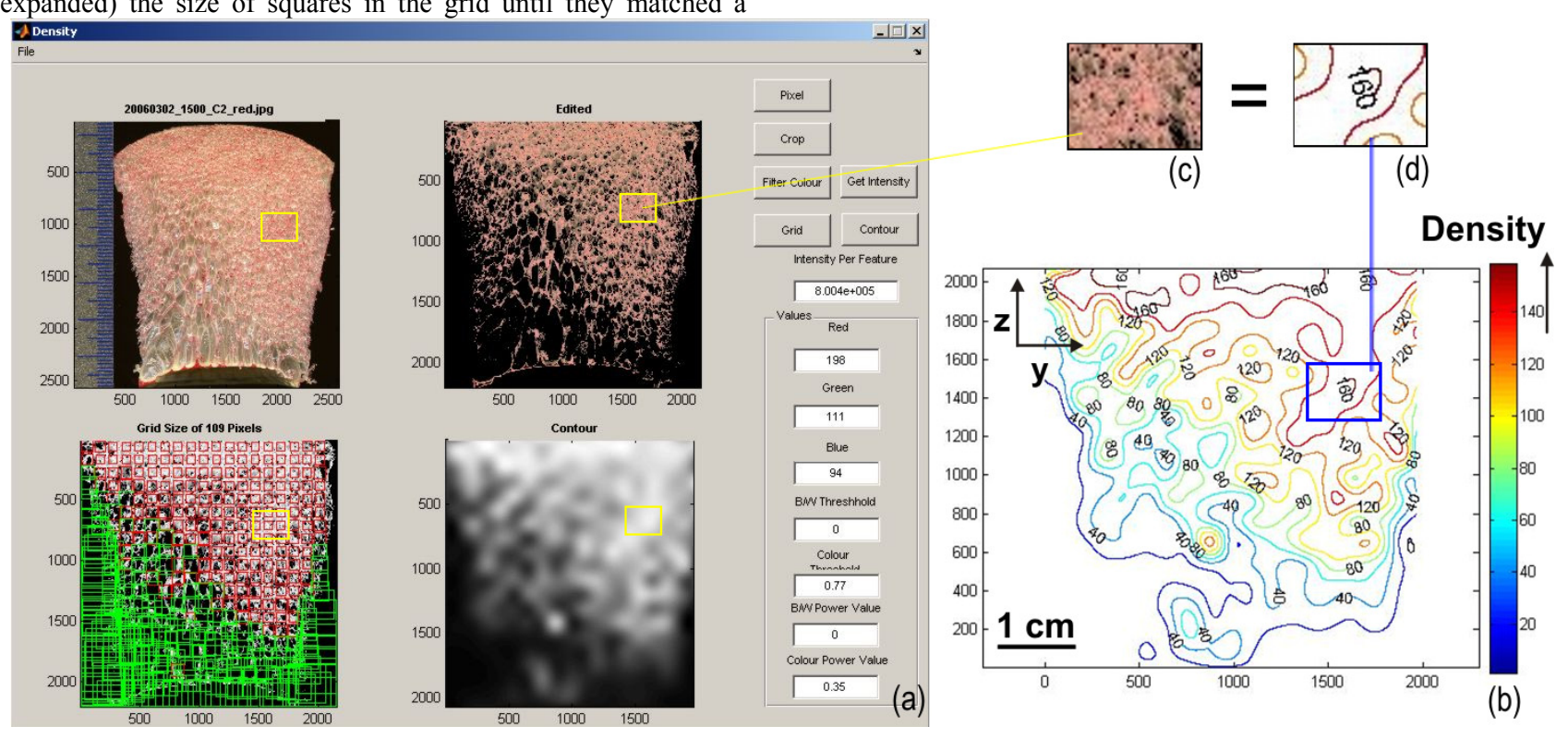

Figure 5: (a) MATLAB ${ }^{\mathrm{TM}}$ Interface; (b) isoporosity contour lines; (c) and (d) correspondent areas in image analysis and contour lines 


\section{RESULTS}

A COMSOL ${ }^{\mathrm{TM}}$ simulation of the experimental environment was used to explore the acoustic field inside of the vessel at each sonication condition (i.e. acoustic pressure and frequency). The values of sound pressure (Figure $6 \mathrm{~b}$ from the COMSOL ${ }^{\mathrm{TM}}$ simulation) and porosity (Figure $6 \mathrm{a}$ from the MATLAB ${ }^{\mathrm{TM}}$ image analysis) along a line through the mid-point (i.e. same depth than the immersed sonotrode's tip) of the container were plotted. This allowed a direct comparison between the porosity gradation measured on the samples' cross-section (Figure 6c) and the acoustic field that they were subjected to. For each frequency of irradiation (i.e. 20,25 and $30 \mathrm{kHz}$ ), the porosity distribution across the section of the foams (solid line) was plotted against the acoustic pressure level in the foam container (as extracted from the COMSOL ${ }^{\mathrm{TM}}$ simulator), assuming the foam's acoustic attenuation to lie between the extremes of water (dash and dot line) and cortical bone (dashed line) (Figure 7). The results suggested that the samples that were irradiated at higher acoustic pressures presented a better correlation between the porosity distribution and the acoustic pressure level. Likewise, those foams irradiated at lower acoustic energy showed a weaker correlation with the simulated pressure distribution.

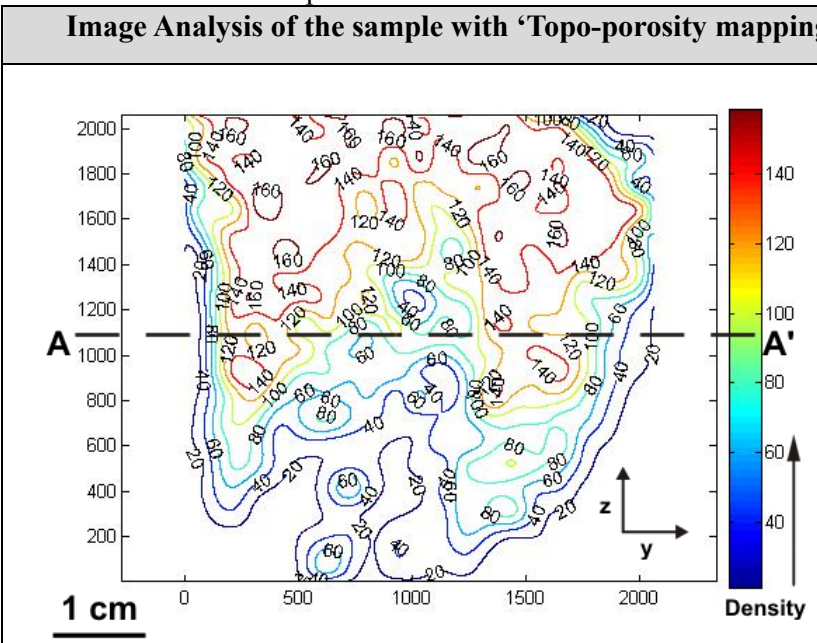

(a) Isoporosity contours from 'Topo-porosity' image analysis program applied to the cross-section of a foam sample
Although the bulk porosity remained approximately the same from early stages of the polymerisation reaction until fully crosslinking of the polymer, the local porosity and, therefore, the acoustic impedance, varied continuously. The acoustic impedance of a viscous fluid is a function of the density of the fluid, its viscosity and the frequency of the ultrasonic wave [27]. During foam cross-linking, the irradiated medium was a mixture of water, carbon dioxide and polyurethane foam. Therefore, the acoustic impedance was expected to change from an initial value similar to water $\left(Z_{\text {water }}=1.48 \mathrm{MRayl}\right)$, through an acoustic impedance similar to resin $\left(Z_{\text {resin }}=1.5-1.8\right.$ MRayl $)$ [28] when the viscosity was high, evolving finally towards values associated with porous materials (7.4-10MRayl) [29] or compact high density bone (9.3MRayl for a density of $1930 \mathrm{~kg} / \mathrm{m}^{3}$ ) [30] when the foam was fully cured and dry. For the purpose of the irradiated foam in the simulated bath, the working acoustic impedances that were used corresponded to the water $\left(\mathrm{Z}=1.48 \mathrm{MRayl}\right.$; density $1000 \mathrm{~kg} / \mathrm{m}^{3}$, longitudinal sound velocity $\mathrm{c}_{\mathrm{S}}=1480 \mathrm{~m} / \mathrm{s}$ ) and to typical trabecular, spongy and wetted interior of bones $\left(\mathrm{Z}_{\text {cort bone }}=2.6 \mathrm{MRayl}\right.$ for a density of $1630 \mathrm{~kg} / \mathrm{m}^{3}, \mathrm{c}_{\mathrm{S}}=1550 \mathrm{~m} / \mathrm{s}$ ) [31], which matched the expected density of the foam at those stages in the reaction.

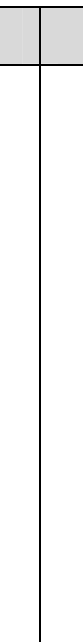

\section{Simulation of the irradiated sample with COMSOL $^{\mathrm{TM}}$}

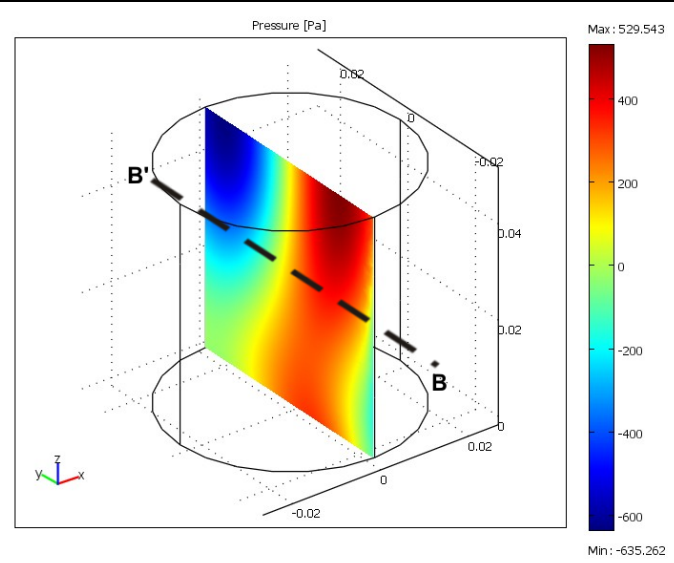

(b) Vertical plane extracted from the COMSOL ${ }^{\mathrm{TM}}$ simulation of the acoustic pressure distribution within the foam vessel immersed in the water bath

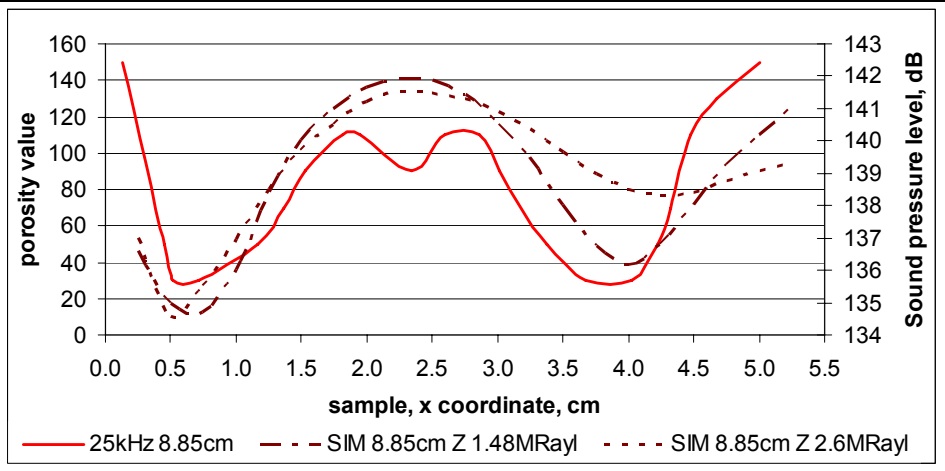

(g) Comparison of experimental porosity values (inverse of density values) extracted along mid-line (AA') of 'sonication plane' aligned with sonotrode vs simulated sound pressure level distribution extracted along mid-line (BB') of the 'sonication plane' aligned with sonotrode for two acoustic impedances $(\mathrm{Z}=1.48 \mathrm{MRayl}$ is water and $\mathrm{Z}=2.6 \mathrm{MRayl}$ is bone) for the irradiated foam

Figure 6: Procedure for analysis of foam irradiated at $25 \mathrm{kHz}$ and $8.85 \mathrm{~cm}$ distant from the sonotrode while immersed in the water bath 


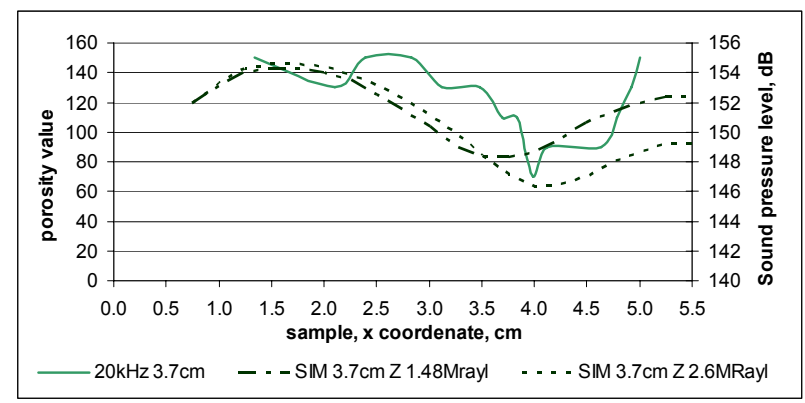

(a)

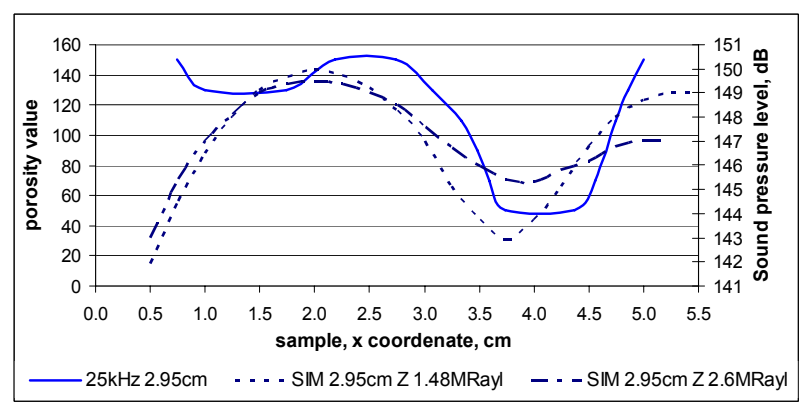

(b)

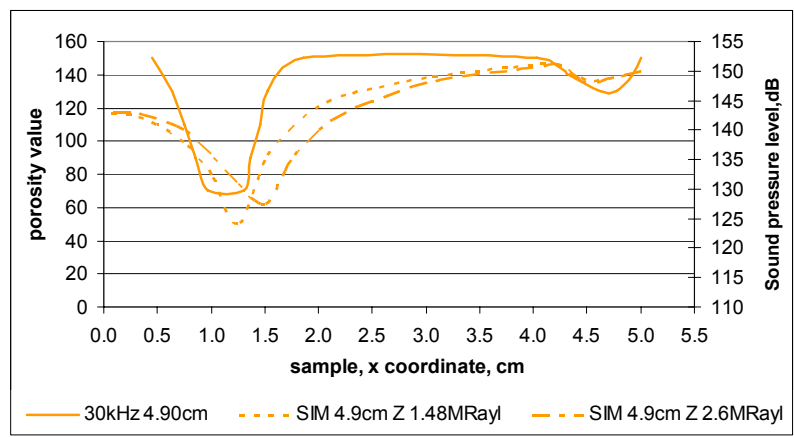

(c)

Figure 7: Comparison of porosity and sound pressure distributions for foams irradiated at the following conditions: (a) $20 \mathrm{kHz}$ and 18 $\mathrm{kPa}$; (b) $25 \mathrm{kHz}$ and $12 \mathrm{kPa}$; (c) $30 \mathrm{kHz}$ and $8.9 \mathrm{kPa}$

\section{DISCUSSION}

The physical phenomena underlying these results can be visualized with a schematic (Figure 8) that illustrates the different ways acoustic cavitation influences the size of bubbles in polymeric foams depending on the level of acoustic pressure. This sketch completes the descriptions of other researchers who have observed situations where gas bubbles submerged in liquid could only suffer enlargement $[32 ; 33]$ (stable cavitation) or implosion

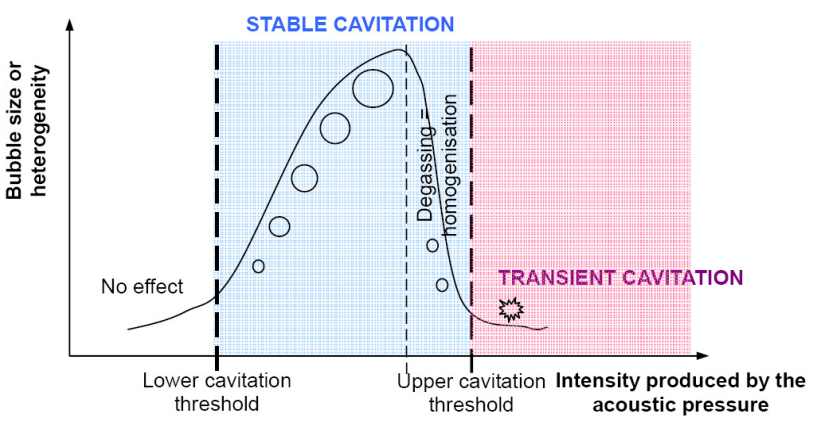

Figure 8: Stages of acoustic cavitation exploited for the tailoring of polymeric foams

The results also offer a valuable insight to the importance of the 'packing' and 'gelation' stages, and the mechanisms that makes them 'sensitive' to ultrasonic irradiation. It is believed that controlled ultrasonic irradiation affects convective mass transfer, especially during rising and packing stages of the foaming process, and enhances the diffusion of the blowing agent (i.e. $\mathrm{CO}_{2}$ gas) from bubble to bubble in the packing and gelation stages. (transient cavitation) [17]. Our results showed that, for the polyurethane foams studied, bubble enlargement was proportional to the sound pressure when this was larger than a lower threshold value (below which there was no effect on the cellular structure), and lower than an upper threshold value, that provoked homogenisation and, at an extreme, collapsing of the polymeric cellular structure through breaking the polymer chains and implosion of bubbles.

\section{CONCLUSIONS}

During the foam polymerisation reaction, the acoustic pressure in the water bath varied causing the bubbles to pulsate in a state of 'stable cavitation' (i.e. rectified diffusion). This pulsation of the bubbles "pumped" gas from the liquid to the gas phase inducing them to increase in volume. The eventual solidification resulted in a porous material with a cellular structure that corresponded with the acoustic field imposed upon it.

The authors conclude that, when conditions of stable cavitation are established, ultrasound can create porosity gradation by producing bubbles of different sizes depending on the acoustic pressure to which they are subjected. This mechanism allows the engineering of standing waves to 'tailor' the porosity of the polymeric matrix that finally solidifies into a foam. In other words, the suitable manipulation of the position of a foaming polymeric melt within a sonication field (with a known acoustic pressure amplitude and frequency), permits the tailoring of the bubbles, and thus the pores, to a desired size. These findings lay the foundations for the development of a multi-source acoustic chamber optimised for the fine control of an acoustic pressure field that could exploit this phenomenon for the manufacturing of tailorable porosity graded materials that can be used in specific 
applications where engineered cellular structures require to be customised (e.g. biomimetics and orthopaedics; structural components, etc).

The effects of ultrasound on porosity demonstrated by this work offer the prospect of a flexible manufacturing process that can control and adjust the cellular geometry of foam and hence ensure that the resulting characteristics of the heterogeneous material match the functional requirements. Further work needs to be done in order to investigate if other polymers' 'sonication window' allows ultrasound to produce a long-lasting effect once the solid structure is set, and if ultrasonic irradiation can also be used as a porosity-tailoring agent when polymers are manufactured under different conditions of pressure, temperature, etc.

\section{REFERENCES}

[1] Mano, J. F., and Reis, R. L., 2005. "Some trends on how one can learn from and mimic nature in order to design better biomaterials." Materials Science and Engineering: C, 25(2), pp. 93-95.

[2] Boccaccini, A. R., 1999. "Fabrication, microstructural characterisation and mechanical properties of glass compacts containing controlled porosity of spheroidal shape." Journal of Porous Materials, 6(4), pp. 369-379.

[3] Seepersad, C. C., Allen, J. K., McDowell, D. L., and Mistree, F., 2008. "Multifunctional Topology Design of Cellular Material Structures." Journal of Mechanical Design, 130(3), pp. 031404-031413.

[4] Peng, H. X., Fan, Z., Evans, J. R. G., and Busfield, J. J. C., 2000. "Microstructure of ceramic foams." Journal of the European Ceramic Society, 20(7), pp. 807-813.

[5] van Tienen, T. G., Heijkants, R. G., Buma, P., de Groot, J. H., Pennings, A. J., and Veth, R. P., 2002. "Tissue ingrowth and degradation of two biodegradable porous polymers with different porosities and pore sizes." Biomaterials, 23(8), pp. 1731-1738.

[6] Chen, P. Y., Lin, A. Y. M., Lin, Y. S., Seki, Y., Stokes, A. G., Peyras, J., Olevsky, E. A., Meyers, M. A., and McKittrick, J., 2008. "Structure and mechanical properties of selected biological materials." Journal of the Mechanical Behavior of Biomedical Materials, 1(3), pp. 208-226.

[7] Sadagopan, D., and Pitchumani, R., 1997. "A Combinatorial Optimization Approach to Composite Materials Tailoring." Journal of Mechanical Design, 119(4), pp. 494-503.

[8] Taboas, J. M., Maddox, R. D., Krebsbach, P. H., and Hollister, S. J., 2003. "Indirect solid free form fabrication of local and global porous, biomimetic and composite 3D polymerceramic scaffolds." Biomaterials, 24(1), pp. 181-194.

[9] Yan, Y., Xiong, Z., Hu, Y., Wang, S., Zhang, R., and Zhang, C., 2003. "Layered manufacturing of tissue engineering scaffolds via multi-nozzle deposition." Materials Letters, 57(18), pp. 2623-2628.

[10] Jackson, T. R., Liu, H., Patrikalakis, N. M., Sachs, E. M., and Cima, M. J., 1999. "Modeling and designing functionally graded material components for fabrication with local composition control." Materials \& Design, 20(2-3), pp. 63-75.

[11] Dutta, D., Prinz, F. B., Rosen, D., and Weiss, L. E., 2001. "Layered Manufacturing: Current status and future trends." Journal of Computing and Information Science in Engineering, 1(1), pp. 60-71.

[12] Gibson, L. J., and Ashby, M. F., 1997. Cellular solids: structure and properties, Cambridge University Press.
[13] Torres-Sánchez, C., and Corney, J., 2008. "Effects of ultrasound on polymeric foam porosity." Ultrasonics Sonochemistry, 15, pp. 408-415.

[14] Zhang, X. D., Macosko, C. W., Davis, H. T., Nikolov, A. D., and Wasan, D. T., 1999. "Role of silicone surfactant in flexible polyurethane foam." Journal of Colloid and Interface Science, 215(2), pp. 270-279.

[15] Rojas, A. J., Marciano, J. H., and Williams, R. J., 1982. "Rigid polyurethane foams: A model of the foaming process." Polymer Engineering \& Science, 22(13), pp. 840-844.

[16] Font, R., Sabater, M. C., and Martínez, M. A., 2002. "The leaching kinetics of acetone in an acetone-polyurethane adhesive waste." Journal of Applied Polymer Science, 85(9), pp. 1945-1955.

[17] Gallego-Juarez, J. A., Rodriguez-Corral, G., RieraFranco De Sarabia, E., Campos-Pozuelo, C., Vazquez-Martinez, F., and Acosta-Aparicio, V. M., 2000. "Macrosonic system for industrial processing." Ultrasonics, 38(1), pp. 331-336.

[18] Gallego-Juarez, J. A., Rodriguez-Corral, G., RieraFranco de Sarabia, E., Vazquez-Martinez, F., Acosta-Aparicio, V. M., and Campos-Pozuelo, C. "Development of industrial models of high-power stepped-plate sonic and ultrasonic transducers for use in fluids." Proceedings of the IEEE Ultrasonics Symposium, 571-578.

[19] Price, G. J., Lenz, E. J., and Ansell, C. W. G., 2002. "The effect of high intensity ultrasound on the synthesis of some polyurethanes." European Polymer Journal, 38(8), pp. 1531-1536. [20] Mason, T. J., Collings, A., and Sumel, A., 2004. "Sonic and ultrasonic removal of chemical contaminants from soil in the laboratory and on a large scale." Ultrasonics Sonochemistry, 11(34), pp. 205-210.

[21] Mulet, A., Carcel, J. A., Sanjuan, N., and Bon, J., 2003. "New food drying technologies - Use of ultrasound." Food Science and Technology International, 9(3), pp. 215-221.

[22] Pitt, W. G., Husseini, G., and Staples, B. J., 2004. "Ultrasonic drug delivery - A general review." Expert Opinion on Drug Delivery, 1(1), pp. 37-56.

[23] Floros, J. D., and Liang, H., 1994. "Acoustically assisted diffusion through membranes and biomaterials." Food Technology, 48(12), pp. 79-84.

[24] Malcolm, A. A., Leong, H. Y., Spowage, A. C., and Shacklock, A. P., 2007. "Image segmentation and analysis for porosity measurement." Journal of Materials Processing Technology, 192, pp. 391-396.

[25] Montminy, M. D., Tannenbaum, A. R., and Macosko, C. W., 2004. "The 3D structure of real polymer foams." Journal of Colloid and Interface Science, 280(1), pp. 202-211.

[26] Torres-Sánchez, C., 2008. "Generation of heterogeneous cellular structures by sonication," Ph.D. thesis, Heriot-Watt University, Edinburgh (UK).

[27] Balasubramaniam, K., and Sethuraman, S., 2006. "Ultrasonic interferometric sensor for rheological changes of fluids." Review of Scientific Instruments, 77(8), pp. 084902084908.

[28] Albers, V. M., 1965. Underwater Acoustics. Handbook $I I$, The Pennsylvania State University Press.

[29] Cheeke, J. D. N., 2002. Fundamentals and applications of ultrasonic waves, CRC publisher.

[30] Raum, K., Cleveland, R. O., Peyrin, F., and Laugier, P., 2006. "Derivation of elastic stiffness from site-matched mineral density and acoustic impedance maps." Physics in Medicine and Biology, 51(3), pp. 747-758. 
[31] Yoshizawa, M., Ushioda, H., and Moriya, T. (2004). "Development of a bone-mimicking phantom and measurement of its acoustic impedance by the interference method." IEEE Ultrasonics Symposium, 1769-1772.

[32] Crum, L. A., 1980. "Measurements of the growth of air bubbles by rectified diffusion." Journal of the Acoustical Society of America, 68(1), pp. 203-211.

of America, 68(1), pp. 203-211.
[33] Leighton, T. G., 1995. "Bubble population phenomena in acoustic cavitation." Ultrasonics Sonochemistry, 2(2), pp. S123-S136. 